\title{
Normal forces exerted upon a long cylinder oscillating in an axial flow
}

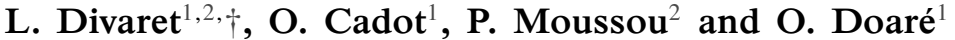 \\ ${ }^{1}$ Unité de Mécanique, Ecole Nationale Supérieure de Techniques Avancées, \\ 828 Boulevard des Maréchaux, 91762 Palaiseau CEDEX, France \\ ${ }^{2}$ LaMSID, UMR CNRS/EDF/CEA 2832, 1 Avenue du Général de Gaulle, 92140 Clamart, France
}

This work aims to improve understanding of the damping induced by an axial flow on a rigid cylinder undergoing small lateral oscillations within the framework of the quasistatic assumption. The study focuses on the normal force exerted on the cylinder for a Reynolds number of $R e=24000$ (based on the cylinder diameter and axial flow velocity). Both dynamic and static approaches are investigated. With the static approach, fluid forces, pressure distributions and velocity fields are measured for different yaw angles and cylinder lengths in a wind tunnel. It is found that for yaw angles smaller than $5^{\circ}$, the normal force varies linearly with the angle and is fully dominated by its lift component. The lift originates from the high pressure coefficient at the front of the cylinder, which is found to depend linearly on the angle, and from a base pressure coefficient that remains close to zero independent of the yaw angle. At the base, a flow deficit and two counter-rotating vortices are observed. A numerical simulation using a $k-\omega$ shear stress transport turbulence model confirms the static experimental results. A dynamic experiment conducted in a water tunnel brings out damping-rate values during free oscillations of the cylinder. As expected from the linear dependence of the normal force on the yaw angle observed with the static approach, the damping rate increases linearly with the axial flow velocity. Satisfactory agreement is found between the two approaches.

Key words: aerodynamics, flow-structure interactions

\section{Introduction}

Fluid forces exerted by an axial flow upon laterally oscillating slender structures are involved in the vibrations of fuel assemblies and heat exchangers in the nuclear industry (Chen 1987; Guo \& Paidoussis 2000), in the stability of towed structures in water (Païdoussis 2004; de Langre et al. 2007), in the behaviour of streamers in the offshore industry (Païdoussis, Price \& de Langre 2011), in fuselage aerodynamics (Bursnall \& Loftin 1951; Hoerner 1985), in animal locomotion (Taylor 1952; Lighthill 1960) and in plant biomechanics (Gosselin \& de Langre 2011). In most of the examples cited above, the fluid forces are expanded in inviscid forces, which are 
(a)

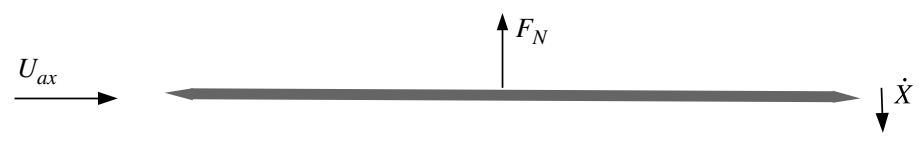

(b)

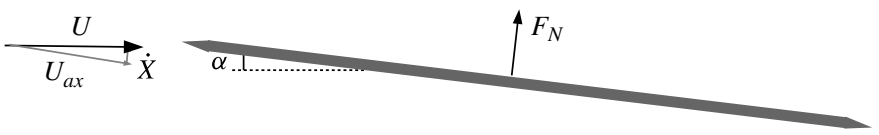

FIGURE 1. (a) Sketch of a slender structure moving with velocity $\dot{X}$ perpendicular to an axial flow of velocity $U$. (b) Sketch of the equivalent problem involving a steady structure in an inclined flow.

obtained purely via potential flow modelling (Lighthill 1960), and drag forces, which are due to more complex fluid-mechanical phenomena such as friction, flow detachment and vortex shedding. The latter forces are generally estimated through empirical models (Morison \& Schaaf 1950; Taylor 1952).

The correct determination of these forces is of major importance in quantifying the flow-induced dissipation on slender structures oscillating perpendicularly to the flow. Using a quasistatic approach, the normal component of the force exerted by the flow on an inclined slender structure can be shown to induce damping. In figure 1 , a slender structure is moving at velocity $\dot{X}$ perpendicular to a main flow with velocity $U$. If transient flow effects due to acceleration of the structure can be neglected, the system is equivalent to a steady solid yawed with a fixed angle of attack, $\alpha=\mathrm{a} \tan (-\dot{X} / U)$, in the same flow. If the oscillation velocity is small compared to the flow velocity, which is often the case in the above applications, the angle of attack becomes small and is equal to the ratio between the velocity of the solid and the axial flow velocity.

Most of the existing literature concerns large yaw angles. The independence principle for yawed cylinders introduced by Relf \& Powell (1917) stipulates that the flow features around a yawed cylinder are determined by the normal component of the velocity; this was first observed experimentally by Relf \& Powell (1917), who showed that the normal force was proportional to the square of the sine of the yaw angle $\alpha$ for $10^{\circ}<\alpha<90^{\circ}$. In the present paper, the yaw angle $\alpha$ represents the angle between the cylinder axis and the axial flow. Hence, the normal force exerted on a yawed cylinder with angle $\alpha$ in a flow of velocity $U$ is equal to that exerted on a cylinder subjected to a cross-flow of velocity $U \sin \alpha$. Theoretical investigations were carried out by Jones (1947) and Sears (1948), who demonstrated that after neglecting small quantities in the laminar boundary-layer equation, the boundary layer of a yawed cylinder in a flow with velocity $U$ is identical to that of a cylinder in cross-flow with velocity $U \sin \alpha$. As the separation point does not depend on the Reynolds number in a two-dimensional laminar flow (Zdravkovich 2003), the independence principle implies that the separation point does not depend on the yaw angle and that the transition to turbulence depends only on the normal component of the velocity. While the independence principle is strictly applicable to a laminar boundary layer, Zdravkovich (2003) highlighted the limitations of this principle with respect to the location of the separation point, as well as the wake of the upstream end. Subsequent experimental investigations have been undertaken by various authors (Bursnall \& Loftin 1951; 
Smith, Kao \& Moon 1971; Ramberg 1983) at large values of the yaw angle (greater than $40^{\circ}$ ). Fewer works are concerned with smaller yaw angles. After observing a significant effect of the skin roughness of marine worms, Taylor (1952) derived a model which takes into account the contribution of the friction force and used the measurements of Relf \& Powell (1917) at intermediate values of the angle. However, no direct measurements supported this representation at that time. In Taylor's model, at small angles the drag force of the cylinder is taken to be constant and equal to its value at $\alpha=0$, where the drag is expected to be mainly due to friction. This model was next used by Hoerner (1965) in an aeronautical context and by De Ridder et al. (2013) for the postprocessing of numerical simulations performed on an oscillating cylinder in axial flow. Complementary values of the friction coefficient were also obtained by Chen (1987) and Païdoussis (2004). In practice, Taylor's model is often modified by introducing two different values of the friction coefficient for the normal and longitudinal directions. The ratio between the two friction coefficients can vary from 0.5 to 2.0 (Ortloff \& Ives 1969) and is based only on roughness considerations.

Recently, Ersdal \& Faltinsen (2006) have carried out both static and dynamic experiments at maximal instantaneous angles between 15 and $30^{\circ}$. They compared the time series of the normal force measured in the dynamic experiments with the time series predicted by a model based on a quasistatic approach. They found a standard error of $15 \%$ in the prediction of the amplitude of the normal force. At lower yaw angles, they observed a linear dependence of the normal force on the yaw angle. Following the approach introduced by Taylor (1952), this linear dependence can be compared to friction effects on the horizontal cylinder. Although sparse, the available experimental values of the normal force coefficient are larger than the value predicted by Taylor's model. Thus, in practice the damping coefficient should be larger than the one predicted by Taylor. As most of the applications mentioned previously concern this range of very low yaw angles, it is of importance to correctly predict the physics of the flow and the behaviour of the normal component of the force in this range. The present article is devoted to this task. The main questions that will be addressed are the following. For a cylinder of circular section, is it valid to consider the drag as representing the main contribution to the normal force, as suggested by Taylor? Is there significant flow detachment at small yaw angles, and what is its influence?

The article is organized as follows: in $\$ 2$, the normal force exerted by the fluid on a yawed cylinder is determined both experimentally and numerically; in $\S 3$, the damping coefficient obtained from a dynamic experiment is compared to the value given by the quasistatic approach; finally, in $\S 4$, the contribution of these results to existing models for the normal force is discussed.

\section{Static approach: cylinder at low yaw angle}

In this section, we present the analysis of the static forces exerted on a yawed cylinder. The experimental set-up is detailed first, and then the experimental results are provided. Numerical simulations have also been performed. The numerical procedure is presented after the description of the experimental set-up, and its results are plotted with the experimental data in all figures. Note that discussion of the similarities and differences between the experimental and numerical results is deferred to the end of the section. 
(a)

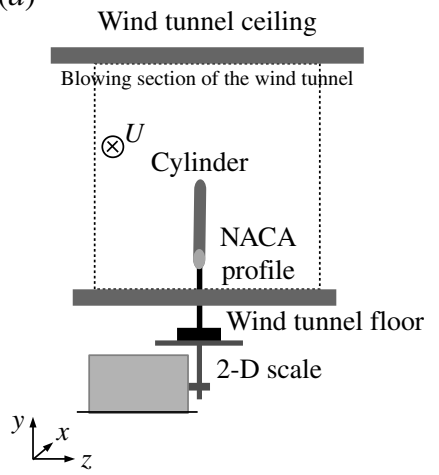

(b)

Wind tunnel ceiling

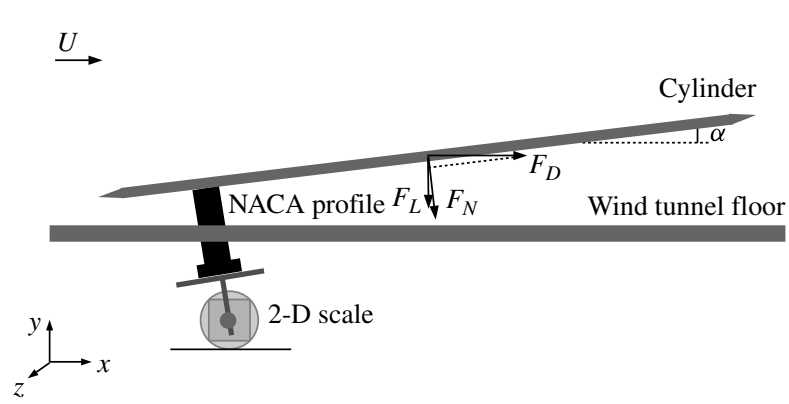

FIGURE 2. Sketch of the set-up for simultaneous drag and lift measurements using a 2-D balance: $(a)$ front view; $(b)$ side view.

(a)

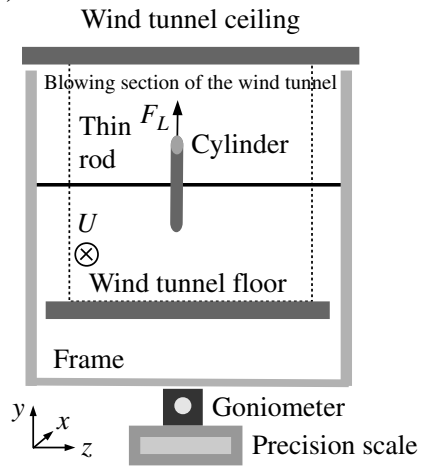

(b)

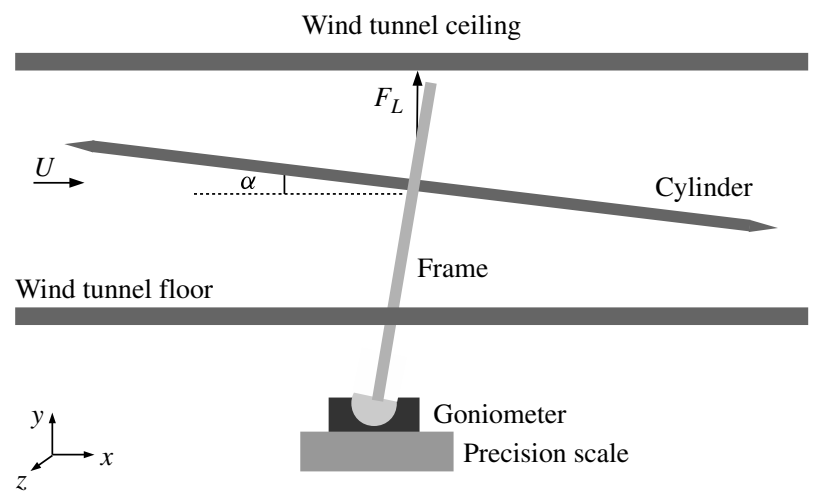

FIGURE 3. Sketch of the set-up for high-accuracy lift measurements: $(a)$ front view; (b) side view.

\subsection{Experimental set-up}

\subsubsection{Geometry}

Experiments are carried out in an Eiffel-type wind tunnel facility. The turbulent intensity is less than $0.3 \%$ and the homogeneity of the velocity over the $400 \mathrm{~mm} \times$ $400 \mathrm{~mm}$ blowing section is $0.4 \%$. The length of the test section is $1.2 \mathrm{~m}$. The models are composed of a cylinder of diameter $D=20 \mathrm{~mm}$ with a cone at both ends with an $11.3^{\circ}$ angle; the length including the ends is $L$. In order to vary the aspect ratio $L / D$ from 15 to 75 , four different lengths are used, $L=0.3,0.6,1.2$ and $1.5 \mathrm{~m}$. The flow velocity $U$ is $18.5 \mathrm{~m} \mathrm{~s}^{-1}$, so that the corresponding Reynolds number based on the cylinder diameter, $R e=U D / v$, is 24000 . Three mounting systems described in figures 2-4 are used to support the cylinder, depending on the measurements to be performed, as discussed below.

\subsubsection{Force measurements}

Due to some constraints imposed by the different force measurements, two different techniques are employed to support the cylinder. 
(a)

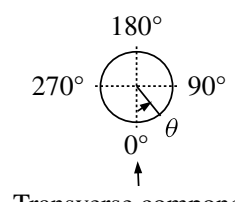

Transverse component of the velocity $U \sin (\alpha)$

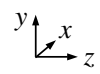

(b)

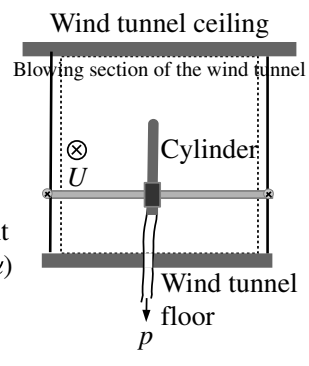

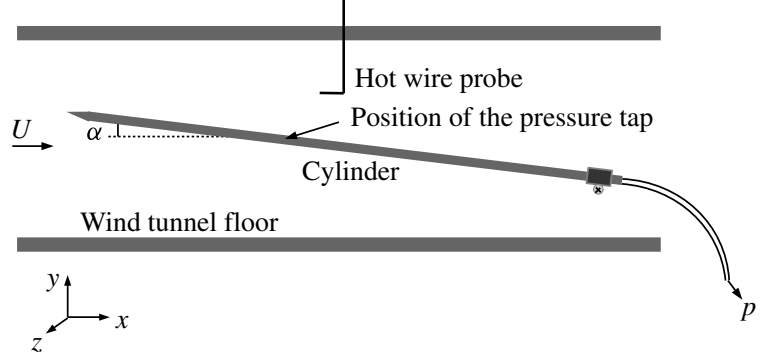

FIGURE 4. Sketch of the set-up for pressure measurements, local velocity measurements and PIV: (a) front view including definition of the azimuthal angle $\theta$ for the pressure measurements; $(b)$ side view.

For simultaneous measurements of the drag $F_{D}$ and the lift $F_{L}$, the cylinder is mounted on a two-component balance via a NACA 0010 profile, as depicted in figure 2. The balance measurement noise is approximately $0.4 \mathrm{~g}$ in lift and $0.6 \mathrm{~g}$ in drag for instantaneous measurements and is equipped with a motorized turntable, allowing inclination of the cylinder with an accuracy of $0.2^{\circ}$. A force measurement corresponds to the time-averaged value obtained by averaging the signal over a period of $120 \mathrm{~s}$ with a sampling frequency of $100 \mathrm{~Hz}$, so that the measurement accuracy is better than $0.3 \mathrm{~g}$. The error bars correspond to the r.m.s. value of the measured force signal. Before each force measurement, a reference measurement is carried out without flow and is used to deduct the contribution of the weight of the system and the balance drift. We applied a force correction due the presence of the NACA profile. The correction is estimated by measuring the force when the cylinder is removed for a zero angle of inclination and under the same flow conditions. The corrections obtained are approximately $0.5 \mathrm{~g}$ for the drag and $-2.0 \mathrm{~g}$ for the lift.

To improve the accuracy of the lift measurements and to prevent the vertical lift produced by the NACA profile, which would degrade measurements at very small yaw angles, the cylinder is held by a frame as shown in figure 3. A high-precision single-component balance $( \pm 0.01 \mathrm{~g})$ sensitive to the lift force supports the frame via a goniometer with a resolution of $0.1^{\circ}$. The two vertical parts of the frame are situated outside the blowing section (see figure $3 a$ ) and do not interfere with the flow. The horizontal part has a circular cylindrical shape of diameter $5 \mathrm{~mm}$ and contributes only to the drag force. As for the two-component balance, a force measurement corresponds to the time-averaged value over a period of $120 \mathrm{~s}$ with a sampling frequency of $1000 \mathrm{~Hz}$. Again, a reference measurement is carried out without flow and used to subtract the contribution of the weight of the system and the balance drift. Because of the vibration of the cylinder, the precision of the balance is altered, and the measurement uncertainty is $0.2 \mathrm{~g}$.

In the following, the force coefficient $C_{i}$ associated with a force $F_{i}$ is defined as

$$
C_{i}=\frac{F_{i}}{\frac{1}{2} \rho U^{2} D L},
$$

where $i=D$ for the drag, $i=L$ for the lift and $i=N$ for the normal force. 


\subsubsection{Pressure measurements}

In order to obtain the pressure distribution $C p(\theta)$ around the cylinder, a third support was designed to allow inclination and rotation of the cylinder, as illustrated in figure 4. The pressure $p$ is measured through a $0.8 \mathrm{~mm}$ hole pierced in the cylinder at $x=0.46 \mathrm{~m}$. The reference pressure $p_{0}$ is measured in the free stream just at the inlet of the test section. A Pitot tube placed upstream of the cylinder provides the dynamic pressure from which the flow velocity $U$ is computed. The pressure taps are connected to a Scanivalve DSA 3217/16px having an accuracy of $\pm 1 \mathrm{~Pa}$. The measurements are averaged over a $3 \mathrm{~min}$ period with an acquisition rate of $500 \mathrm{~Hz}$. The standard deviation of the pressure time series is used to estimate the uncertainty of the measurement. The pressure coefficient is defined as

$$
C_{p}=\frac{p-p_{0}}{\frac{1}{2} \rho U^{2}}
$$

Finally, to realize a pressure distribution $C_{p}(\theta)$, the cylinder is rotated by increments of $15^{\circ}$ with a precision of $3^{\circ}$.

\subsubsection{Velocity measurements}

Hot-wire probe. Local velocity measurements are performed using a single hot-wire probe from DANTEC (hot-wire type 55P15, support type 55H22). It is connected to a DISA55 hot-wire anemometer with an overheat ratio of 1.5. The probe is mounted on a traversing system placed on the ceiling of the test section to allow a displacement orthogonal to the cylinder axis when it is horizontal $\left(\alpha=0^{\circ}\right)$. The wire is oriented in such a way as to be sensitive to the modulus of the velocity, denoted by $u$ in the plane $(x, y)$. The displacement allows the acquisition of a velocity profile, on the upper part of the cylinder only $(y>0)$, at a distance of $x=0.5 \mathrm{~m}$ from the upstream end of the cylinder. The value $U$ of the velocity is averaged over $30 \mathrm{~s}$ with a sampling rate of $1 \mathrm{kHz}$. The boundary-layer thickness $\delta_{99}$ and the displacement thickness $\delta_{1}$ are deduced from the velocity profile measured with the hot-wire probe using the following equations:

$$
\begin{gathered}
U_{D}\left(\delta_{99}\right)=0.99 U_{D \infty}, \\
\delta_{1}=\frac{1}{U_{D \infty}} \int_{0}^{\infty}\left(U_{D \infty}-U_{D}\right) \mathrm{d} y .
\end{gathered}
$$

Particule image velocimetry. Wake visualizations are performed using particle image velocimetry (PIV). The system is composed of a DANTEC dual pulse laser (Nd:YAG, $2135 \mathrm{~mJ}, 4 \mathrm{~ns}$ ) and two DANTEC CCD cameras (FlowSense EO, 4 Mpx). The device acquires image pairs at a rate of $10 \mathrm{~Hz}$; each acquisition records 2000 image pairs. The interrogation window size is $32 \times 32$ pixels with an overlap of $25 \%$. The stereo PIV measures the three components of the velocity in the $(y, z)$ plane at the same location along the cylinder as the pressure measurements. The $32 \times 32$ pixels of the interrogation window correspond to physical sizes of $2.4 \mathrm{~mm} \times 2.4 \mathrm{~mm}$. The mean velocity and vorticity fields are computed from the valid vectors of the 1000 measurements. 


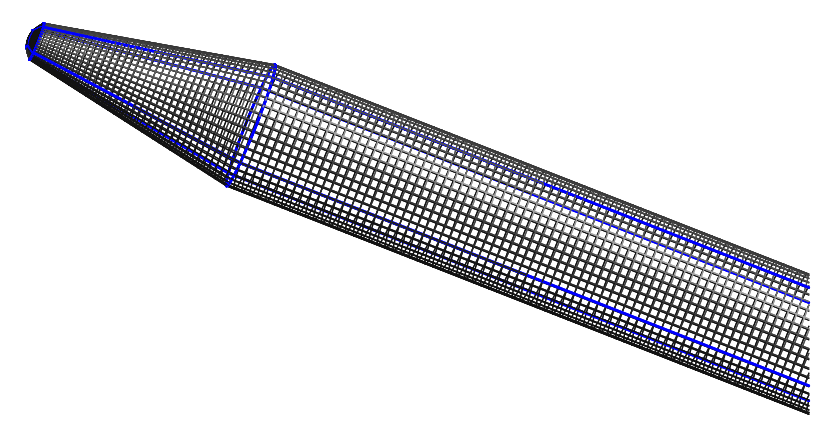

FIGURE 5. (Colour online) Detail of the mesh on the cylinder surface.

\subsection{Numerical method}

All the numerical results presented in this study were performed with the finite-volume code Code_Saturne described in Archambeau, Sakiz \& Namane (2004). The fluid volume is discretized with a conform quadrangle mesh obtained with the mesh generator gmsh developed by Geuzaine \& Remacle (2009). The characteristic length of the cells for the reference mesh is $\Delta x=1.6 \mathrm{~mm}$. The mesh is made up of 16 million cells. A time- and space-varying time step with characteristic value $\Delta t=0.0001 \mathrm{~s}$ is applied in order to fulfil the CFL condition $\Delta t \sum_{i=1}^{3}\left(v_{i}\right) /\left(\Delta x_{i}\right)<1$ for every cell of the mesh. The code uses a centred scheme for the velocity and a simplec algorithm for the velocity-pressure coupling. The simulations performed are RANS simulations with the shear stress transport (SST) $k-\omega$ turbulence model of Menter (1993, 1994). Due to the relatively high Reynolds number, a second-order wall law is used, and the cells close to the cylinder are large enough to ensure that $y^{+}>50$.

The dimension of the fluid domain is $0.4 \mathrm{~m} \times 0.4 \mathrm{~m} \times 1.2 \mathrm{~m}$, i.e. the same as the wind tunnel test section. The size of the cylinder is also the same as in the experiments: the diameter of the cylinder is $D=0.02 \mathrm{~m}$ and the reference length-todiameter ratio is $L / D=0.6$. Simulations were also performed on cylinders with $L / D=$ 0.15 and 0.3 . The ends of the cylinder are slightly different in the numerical case: the last $20 \%$ of the cones at the ends of the cylinder are replaced with a spherical cap (see figure 5).

A constant and uniform velocity is imposed at the inlet and a constant static pressure imposed at the outlet. The sides of the domain have slip wall conditions (only the normal velocity is equal to zero at the boundary), and the cylinder has a wall boundary condition (see figure 6). Simulations are performed for $L / D=0.6$ with yaw angles $\alpha=0,1,2,3,3.6,4,5,6.3$ and $8.3^{\circ}$ and for $L / D=0.15$ and 0.30 with $\alpha=0,2$ and $4^{\circ}$.

Other numerical simulations have been performed with a coarser mesh $(\Delta x=$ $2.6 \mathrm{~mm}$ ). The results are similar to those obtained using the more refined mesh, except for yaw angles $\alpha>6^{\circ}$, for which the pressure distribution is affected but the force coefficients are still accurate. Only the results obtained in the reference case with $\Delta x=1.6 \mathrm{~mm}$ are presented in this paper.

\subsection{Results}

\subsubsection{Flow characterization}

At the horizontal position of the cylinder $\left(\alpha=0^{\circ}\right)$, a boundary layer develops along the cylinder and creates a velocity deficit region. A velocity profile has been 


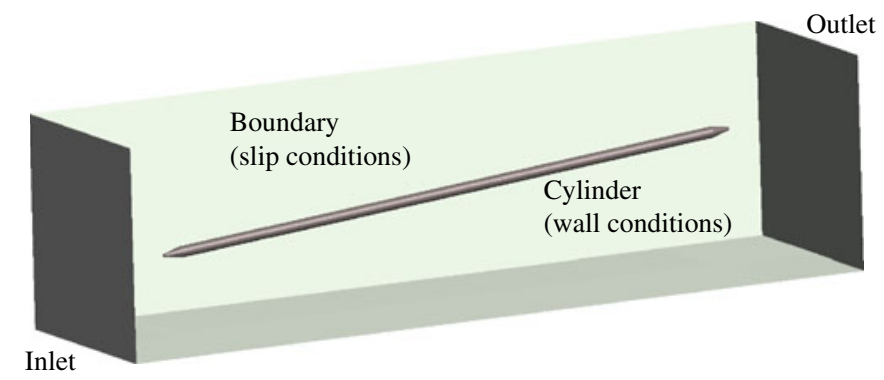

FIGURE 6. (Colour online) Boundary conditions for the numerical simulations.

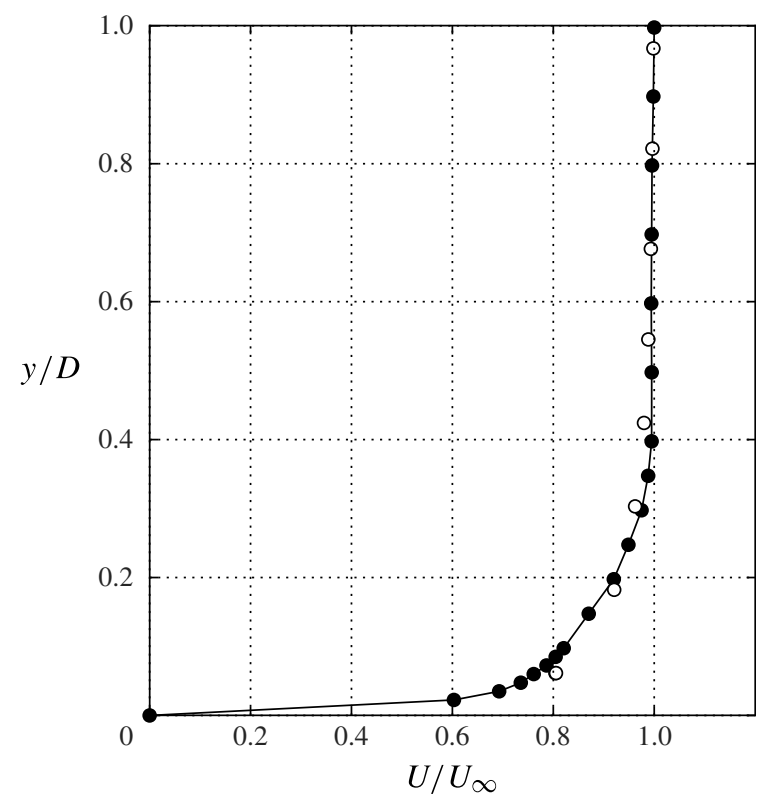

FIGURE 7. Velocity $U$ plotted against distance $y$ to the cylinder at the position located $0.5 \mathrm{~m}$ from the upstream end of the cylinder, for the case where the cylinder is horizontal $\left(\alpha=0^{\circ}\right), U_{\infty}=18.5 \mathrm{~m} \mathrm{~s}^{-1}$ and $R e=24000$. The filled circles represent experimental points and the empty circles simulation results.

measured at the middle position $x=0.5 \mathrm{~m}$ (see figure 4), at $R e=24000$. The boundary-layer thickness and the displacement thickness are calculated from the experimental velocity profile, where $y=0$ corresponds to the cylinder surface; see the filled circles in figure 7 . The boundary-layer thickness is $\delta_{99}=0.4 D$, and the corresponding displacement thickness is estimated to be $\delta_{1} / D=0.06$.

\subsubsection{Force measurements and finite-length effects}

The normal force exerted by the fluid on the cylinder has a contribution due to the drag and a contribution due to the lift:

$$
C_{N}=C_{L} \cos \alpha+C_{D} \sin \alpha .
$$




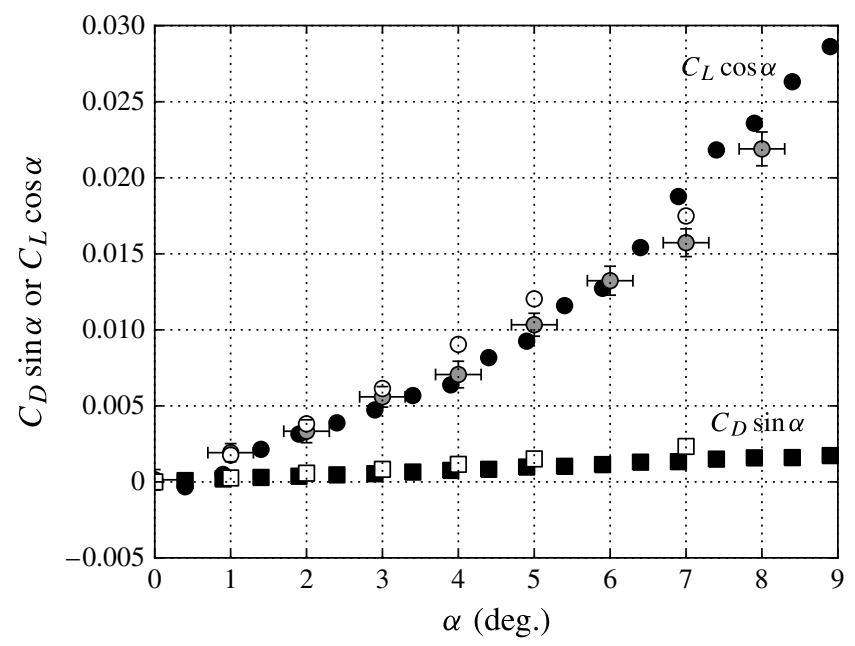

FIGURE 8. Normal components of the drag force $C_{D} \sin \alpha$ (squares) and lift force $C_{L} \cos \alpha$ (circles), as defined in (2.5), plotted against the yaw angle $\alpha$ at $R e=24000$. Filled symbols are experimental results and empty symbols numerical simulations. Black symbols refer to the 2-D balance and grey symbols to the precision-scale measurements as depicted in figures 2 and 3.

These contributions are obtained with the two-component balance as shown in figure $1(a)$. Measurements for yaw angles from 0 to $9^{\circ}$ in increments of $0.5^{\circ}$ are presented in figure 8 (filled symbols) and show that the drag contribution is always much smaller than the lift contribution, even for yaw angles smaller than $5^{\circ}$. A linear fit of the experimental data gives $C_{D}=0.012 \pm 0.001$. This value is in agreement with the range of values $0.008<C_{D}<0.020$ given by Païdoussis (2004). Other measurements performed at $15 \mathrm{~m} \mathrm{~s}^{-1}(R e=19500)$ and $25 \mathrm{~m} \mathrm{~s}^{-1}(R e=32500)$ yield similar results. From now on, we will restrict our attention to the lift force. For this purpose, a single-component precision balance as depicted in figure 3 is used. The lift obtained is represented by the grey circles in figure 8 and agrees well with previous measurements performed with the two-component balance. The lift coefficients for positive and negative yaw angles $\alpha$ are shown in figure 9 and compared with the lift coefficient $C_{L}^{\text {ind }}$ given by the independence principle,

$$
C_{L}^{\text {ind }}=C_{D_{\alpha=90^{\circ}}} \sin ^{2} \alpha \cos \alpha,
$$

where $C_{D_{\alpha=90^{\circ}}}$ is the experimental cross-flow drag coefficient at $R e=24000$ measured in an additional experiment. Two different kinds of behaviour can be observed: a quadratic relationship for angles $|\alpha|>5^{\circ}$ (figure $9 a$ ) and a linear one for $|\alpha|<5^{\circ}$ (figure $9 b$ ). The quadratic law of the independence principle is in agreement with the quadratic variation of the measurements for large $\alpha$. Obviously the independence principle does not hold for $\alpha<5^{\circ}$.

In order to estimate the contribution of the cylinder ends to the lift, we repeated measurements for four cylinder lengths, $L / D=15,30,60$ and 75 , and yaw angles $|\alpha|<5^{\circ}$. As shown in figure 10(a), for all cylinders, the lift coefficient is proportional to the inclination. The mean slope is deduced by taking an average of the upper and lower bounding lines that enclose the data: the slope is the average of the slopes of the 

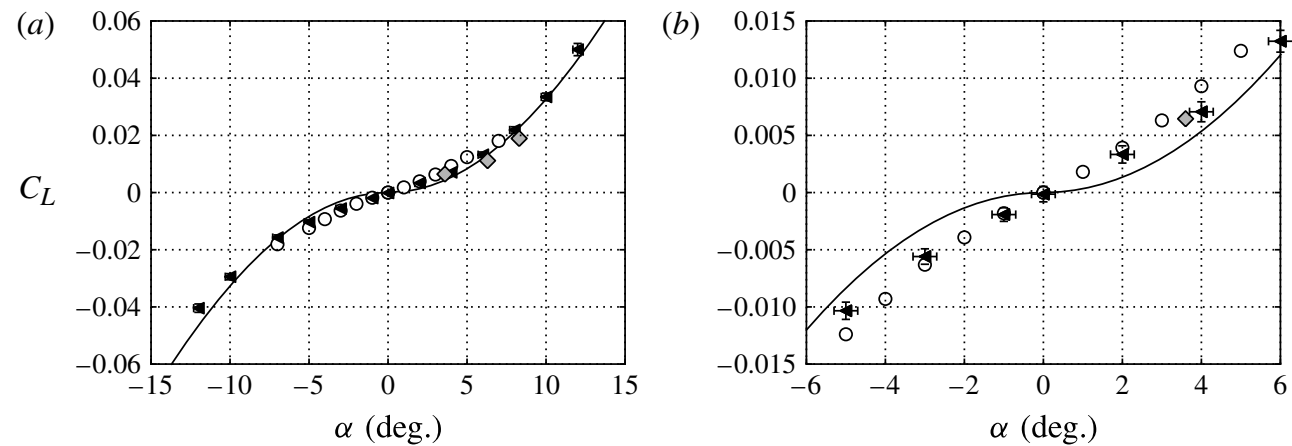

FIGURE 9. (a) Experimental (filled symbols) and numerical (empty symbols) lift coefficients $C_{L}$ plotted against the yaw angle $\alpha$ for $L / D=60$ at $R e=24000 ;(b)$ zoomed-in plot for low yaw angles. In both panels, black symbols represent lift coefficients measured with the precision balance, while grey symbols represent lift coefficients $C_{L_{p}}$ obtained by integration of the pressure distributions (see figure 11); the solid curves represent the lift coefficient given by the independence principle.
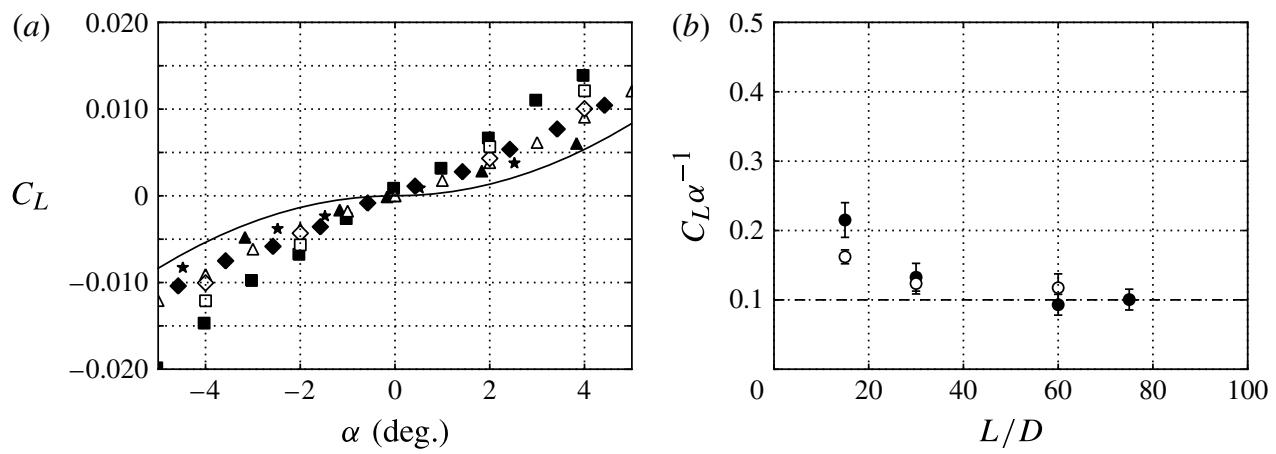

FIGURE 10. (a) Experimental (filled symbols) and numerical (empty symbols) lift coefficients $C_{L}$ plotted against the inclination $\alpha$ at $R e=24000$, for different length-todiameter ratios: $L / D=15$ (squares), 30 (diamonds), 60 (triangles) and 75 (stars); the solid curve represents the lift coefficient given by the independence principle. (b) Experimental (filled symbols) and numerical (empty symbols) slopes of the lift coefficient plotted against the length-to-diameter ratio; the dashed horizontal line represents the asymptote for $L / D>60$.

lines and the uncertainty is their difference. The slope $C_{L} \alpha^{-1}$ is shown in figure $10(b)$; the lift is larger for smaller cylinders, indicating that effects of the ends induce an additional lift. For $L / D>60$ the lift coefficient slope remains constant, implying that the contribution of the ends becomes negligible. Focusing only on the long-cylinder limit, from these results we can deduce a general law for the normal force coefficient, of the form

$$
C_{N}=C \alpha \quad \text { with } C=0.11 \pm 0.016, \quad \text { for }|\alpha|<5^{\circ} \text {. }
$$

This value is in the same range as the $C$ value 0.068 found by Ersdal \& Faltinsen (2006) in their experiments performed on cylinders with aspect ratios $L / D=31.25$ and 10.48 . 
Moreover, flow velocities ranging from $13(R e=17000)$ to $25 \mathrm{~m} \mathrm{~s}^{-1}(R e=32500)$ have been tested, yielding similar results. The corresponding damping coefficient $C$ for $\alpha<5^{\circ}$ will be presented in figure 20 .

We recall here the two main results that have emerged at this stage of the investigation. Firstly, the contribution of the drag force is small compared to that of the lift force (figure 8). Second, at small yaw angles, the normal force coefficient is proportional to the yaw angle, as in (2.7). The coefficient found in the present analysis is then higher than that found using only the drag contribution (Taylor 1952). In the following, $\S \S 2.3 .3$ and 2.3.4 are devoted to analysis of the pressure distribution and characterization of the wake in small-angle regimes.

\subsubsection{Pressure distributions}

The angular distributions of the pressure $C_{p}(\theta)$ are shown in figure 11 for yaw angles $\alpha=3.6,6.3,8.3$ and $21^{\circ}$. These results are all compared to the pressure distribution deduced from the independence principle,

$$
C_{p_{\alpha}}^{\text {ind }}(\theta)=C_{p_{\alpha=90^{\circ}}}(\theta) \sin ^{2} \alpha,
$$

where $C_{p_{\alpha=90^{\circ}}}$ is the experimental cross-flow distribution at $R e=24000$ coming from the same additional experiment as for $C_{D_{\alpha=90^{\circ}}}$ in (2.6). According to figure 11, the pressure distributions do not follow the independence principle for small yaw angles. The expression in (2.8) implies a flow separation at $80^{\circ}$, which is certainly not the case for small angles, because the elliptical shape seen by the flow introduces weaker adverse pressure gradients than that of a circular shape. Thus it is expected that the independence principle does not hold around $\theta=80^{\circ}$. On the other hand, it is surprising to find disagreement at the dividing streamline at $\theta=0^{\circ}$. The pressure prediction from the independence principle is always lower than the measured value of $C_{p}\left(\theta=0^{\circ}\right)$. Only for $\alpha=21^{\circ}$ is the pressure coefficient before the separation in accordance with the independence principle, which is consistent with the results in the literature for larger yaw angles (Bursnall \& Loftin 1951).

For each pressure distribution shown in figure 11, the sectional normal coefficient is computed by the formula

$$
C_{N_{p}}=\oint_{0}^{360} C_{p}(\theta) \cos \theta \mathrm{d} \theta .
$$

The lift coefficient is then defined as $C_{L_{p}}=C_{N_{p}} \cos \alpha$. The values obtained are displayed as grey diamonds in figure 9 . They retrieve the lift obtained by the balance measurements. This agreement indicates that the pressure distribution does not vary much along the cylinder as would be expected from the previous study on cylinder length shown in figure $10(b)$. The fact that the independence principle is not well observed in the pressure distribution at $\alpha=8.3^{\circ}$ does not contradict the corresponding lift coefficient that follows the quadratic dependence in figure 9; this is due to the integration effect in (2.9), which is not sensitive to the pressure distribution in the vicinity of the cylinder sides. In other words, the normal force is mainly due to the pressure difference between the front and the base of the cylinder, and the pressure at intermediate angles plays only a minor role. More extensive measurements have been performed at $\theta=0$ and $180^{\circ}$, as shown in figure 12. The front pressure coefficient $C_{p}\left(\theta=0^{\circ}\right)$ exhibits the same types of variation with the yaw angle as the lift force: it is linear at small angles and quadratic at higher angles. For the base pressure 

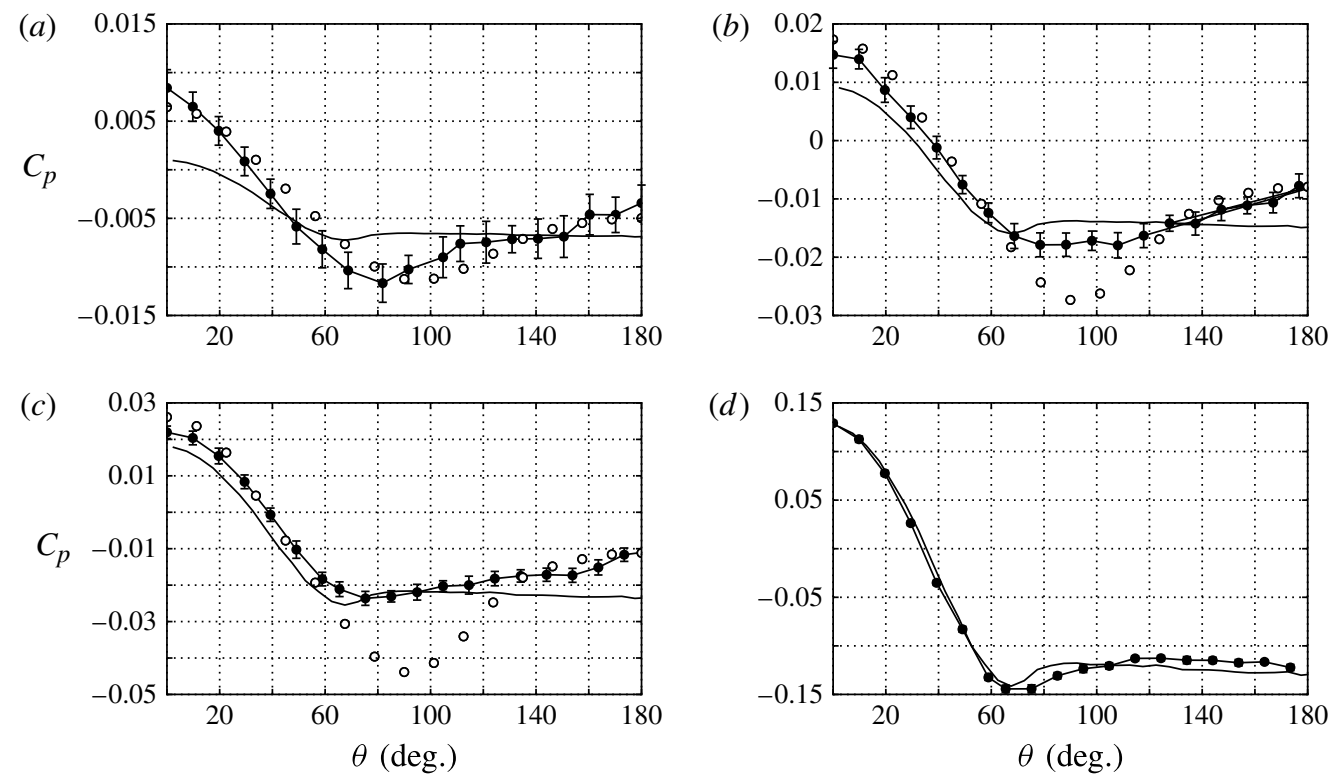

FIGURE 11. Experimental (filled symbols) and numerical (empty symbols) pressure coefficients $C_{p}$ plotted against the angle $\theta$ at $R e=24000$, for four inclination angles: (a) $\alpha=3.6^{\circ} ;(b) \alpha=6.3^{\circ} ;(c) \alpha=8.3^{\circ} ;(d) \alpha=21.0^{\circ}$. In each panel, the thin black curve represents the pressure coefficient given by the independence principle.

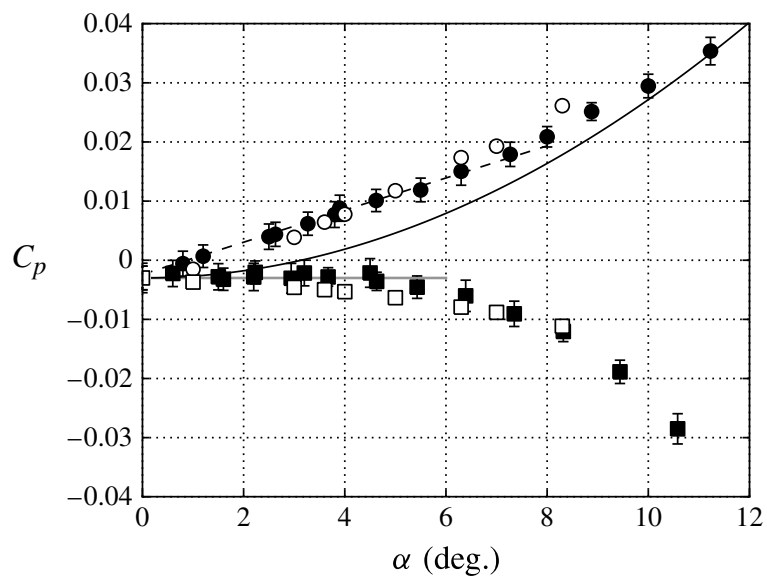

FIGURE 12. Experimental (filled symbols) and numerical (empty symbols) pressure coefficients at the front (circles) and the base (squares) of the cylinder at $x=0.46 \mathrm{~m}$ plotted against the inclination $\alpha$. The solid black curve represents the front pressure given by the independence principle; the dashed line shows the linear regression $C_{p}=$ $0.16 \alpha$ (rad) for the front pressure, for $\alpha<5^{\circ}$; the grey line indicates $C_{p}$ at $\alpha=0^{\circ}$.

$C_{p}\left(\theta=180^{\circ}\right)$, the behaviour is different: the pressure is constant for yaw angles lower than $\alpha=5^{\circ}$ and decreases quadratically beyond. The slightly negative value of $C_{p}=-0.0037$ at $\alpha=0^{\circ}$ is merely a consequence of the obstruction of the uniform flow by the cylinder. From these results, it can be deduced that the linear variation of 
the lift force with yaw angles in the range $|\alpha|<5^{\circ}$ is governed by a linear variation of the front pressure together with a constant base pressure.

\subsubsection{Wake characterization}

PIV measurements have been carried out in the $(y, z)$ plane at the same location along the cylinder as the pressure measurements. The velocity field in the $x$ direction at the back of the cylinder is presented in figure 13. For the horizontal position of the cylinder, i.e. $\alpha=0^{\circ}$, figure 13(a) shows an axisymmetric velocity deficit close to the cylinder, which corresponds to the boundary layer. Its thickness corresponds to the one of $0.4 \mathrm{D}$ measured with the hot-wire probe (figure 7 ). The wake has a two-lobe shape and its length increases with the yaw angle, as can be seen in figure $13(b-d)$. The vorticity field in the $x$ direction, $\omega_{x}=\partial v_{z} / \partial y-\partial v_{y} / \partial z$, is plotted in figure 14 and shows two stationary vortices. Their presence reveals a three-dimensional separation along the cylinder even at low yaw angles $|\alpha|<5^{\circ}$. Comparison with the velocity field in figure 13 indicates that the cores of the vortices are associated with a strong velocity deficit.

\subsection{Comparison of experimental and numerical results}

For comparison's sake, the results of the numerical simulations have been systematically compared with experimental results in all the previous figures. The velocity field was characterized first for the horizontal position of the cylinder. Figure 7 shows that at $\alpha=0^{\circ}$, the boundary layer is well represented by the numerical simulations.

The forces obtained numerically are very close to those measured in the experiments. In figure 8 , the numerical and experimental drag and lift components of the normal force have close values, and the result about the drag component slightly contributing to the normal force is reproduced numerically. As for the experiments, the numerical lift coefficient shown in figure $9(a)$ varies linearly with the yaw angle for $|\alpha|<5^{\circ}$, and quadratically for $|\alpha|>5^{\circ}$. The slopes in the linear range shown in figure $9(b)$ differ slightly: $C_{L} \alpha^{-1}$ is $0.1 \pm 0.015$ in the experiments and $0.12 \pm 0.020$ in the numerical simulations (figure 10). The numerical lift coefficients decrease with the length of the cylinder and their slopes converge for sufficiently long cylinders, which is in agreement with the experimental results.

For the pressure distributions shown in figure 11, comparison of the numerical and experimental pressure distributions shows that the numerical simulations represent well the regions $0^{\circ}<\theta<60^{\circ}$ and $130^{\circ}<\theta<180^{\circ}$. In the region $60^{\circ}<\theta<130^{\circ}$, the numerical pressure coefficient is lower than the experimental one. Figure 12 shows both numerical and experimental pressure coefficients at $\theta=0$ and $180^{\circ}$ for different yaw angles. The numerical and experimental results are in good agreement: the pressure coefficient at $\theta=0^{\circ}$ varies linearly with the yaw angle until $\alpha=5^{\circ}$, whereas the pressure coefficient $\theta=180^{\circ}$ is almost constant.

Figures 15 and 16 represent the velocity and vorticity fields in the incoming flow direction at yaw angles $\alpha=0,3.6,6.8$ and $8.3^{\circ}$, and can be directly compared with figures 13 and 14. The region of small axial velocity is larger by a factor of 2 in the numerical simulations, except for the yaw angle $\alpha=0^{\circ}$. The footprint of the two counter-rotating vortices is not visible in the deficit region, probably because they are weaker by approximately a factor of 10 (see figure 16). 

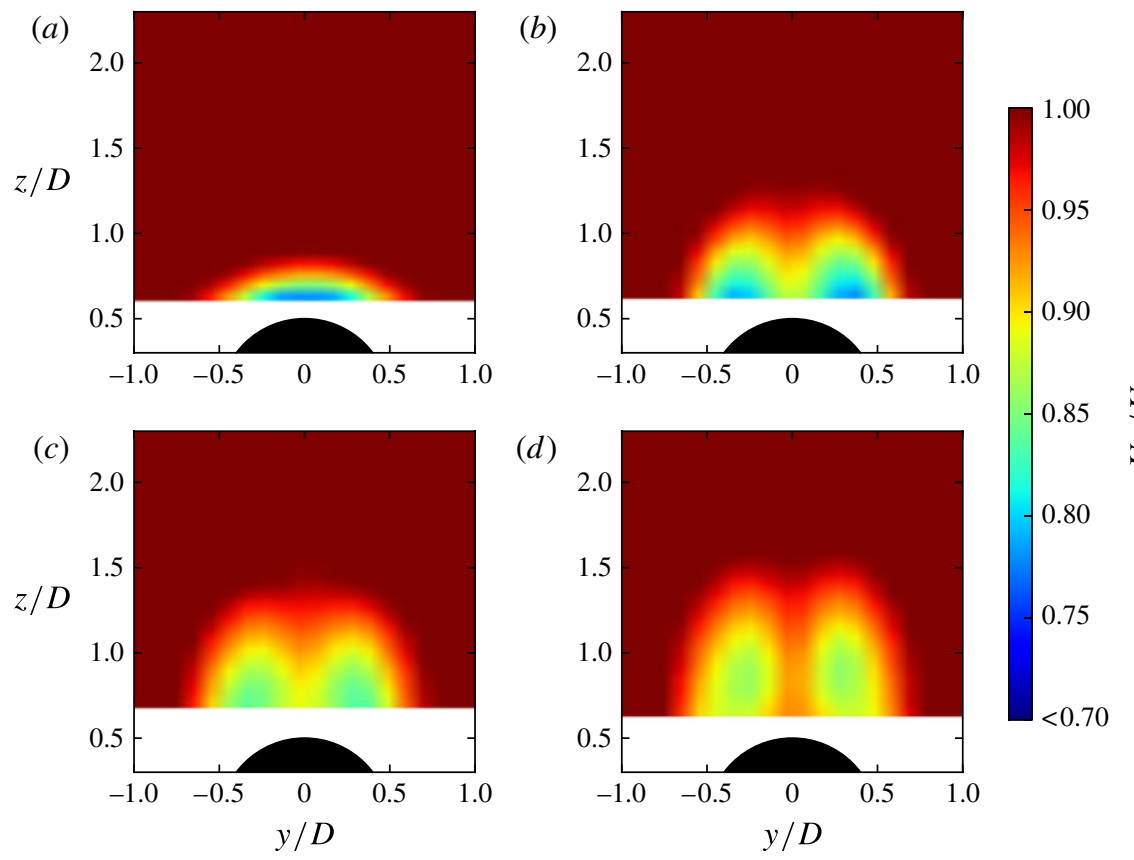

FIGURE 13. Experimental velocity in the $x$ direction at the back of the cylinder for four yaw angles: (a) $\alpha=0^{\circ}$; (b) $\alpha=3.6^{\circ} ;$ (c) $\alpha=6.3^{\circ} ;$ (d) $\alpha=8.3^{\circ}$.
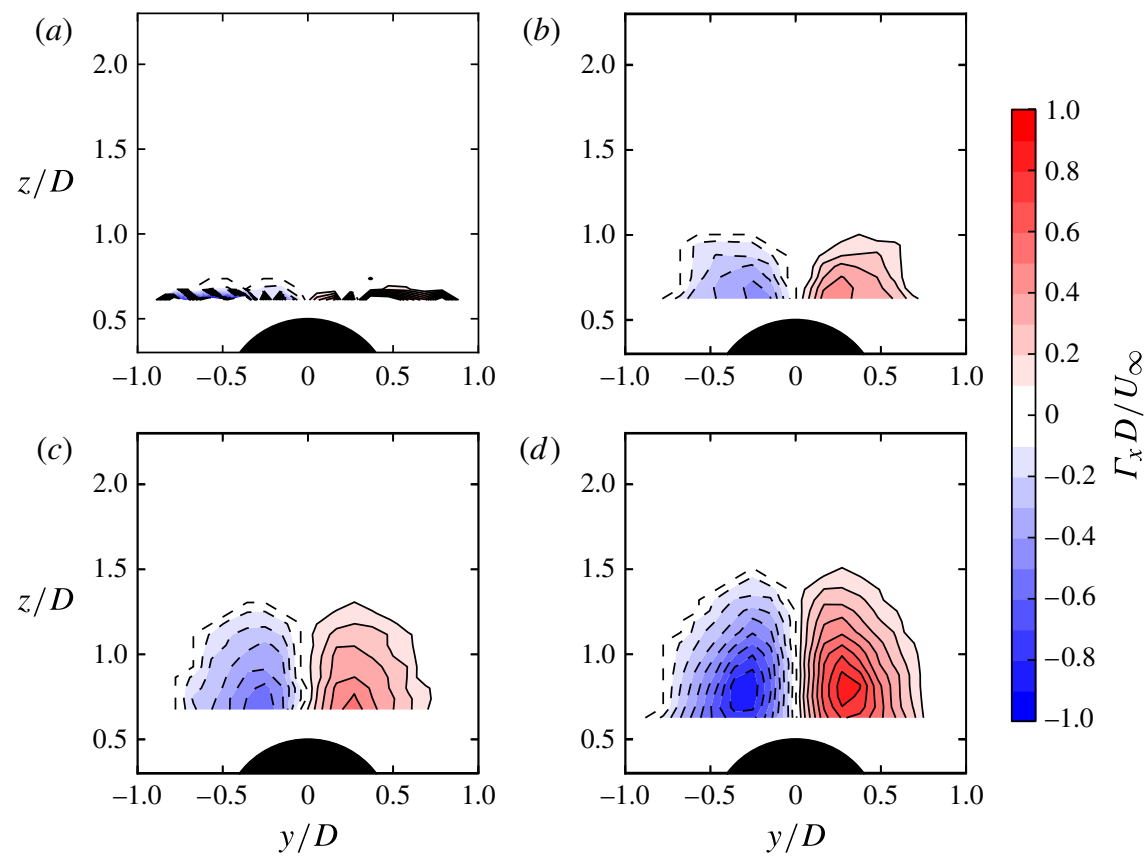

FIGURE 14. Experimental vorticity in the $x$ direction at the back of the cylinder for four yaw angles: (a) $\alpha=0^{\circ}$; (b) $\alpha=3.6^{\circ}$; (c) $\alpha=6.3^{\circ} ;(d) \alpha=8.3^{\circ}$. The black lines represent isolines of positive vorticity, and the dashed lines represent isolines of negative vorticity (in steps of 0.1 ). 

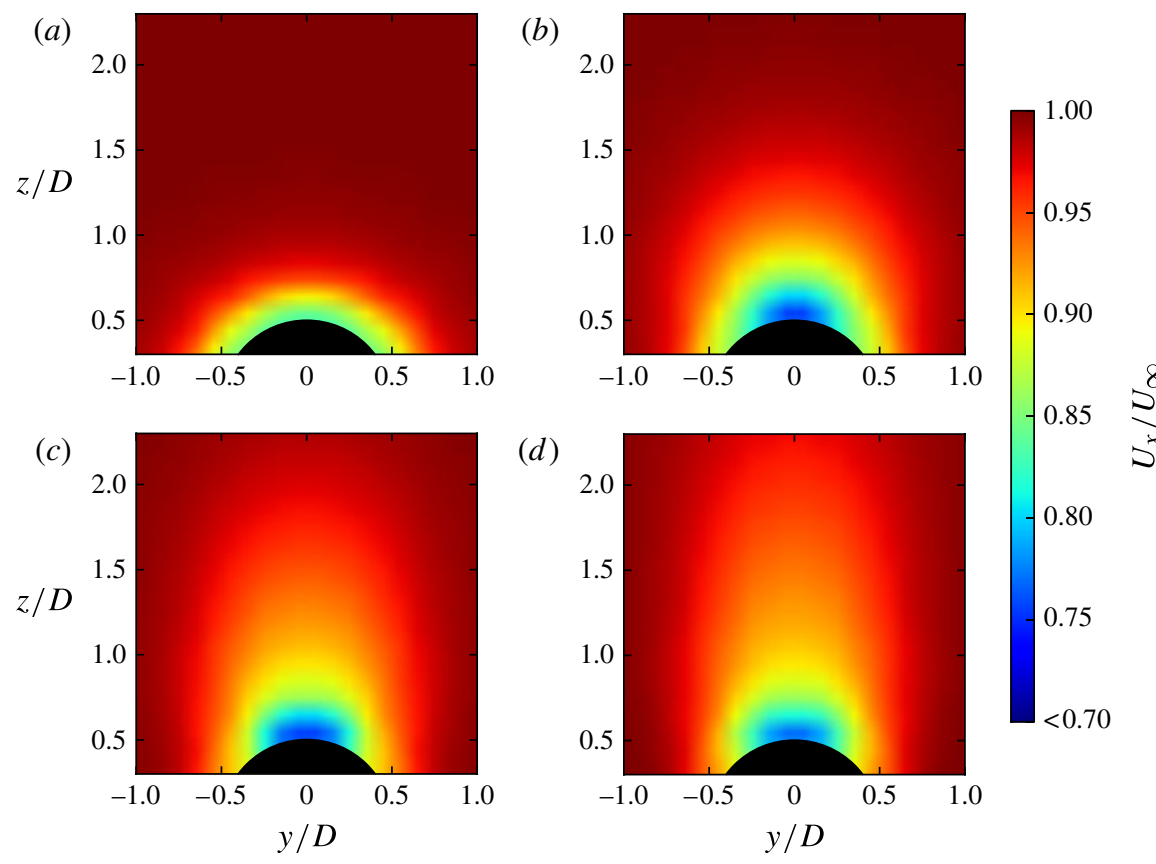

FIGURE 15. Numerical velocity in the $x$ direction at the back of the cylinder for four yaw angles: (a) $\alpha=0^{\circ}$; (b) $\alpha=3.6^{\circ} ;$ (c) $\alpha=6.3^{\circ}$; (d) $\alpha=8.3^{\circ}$.
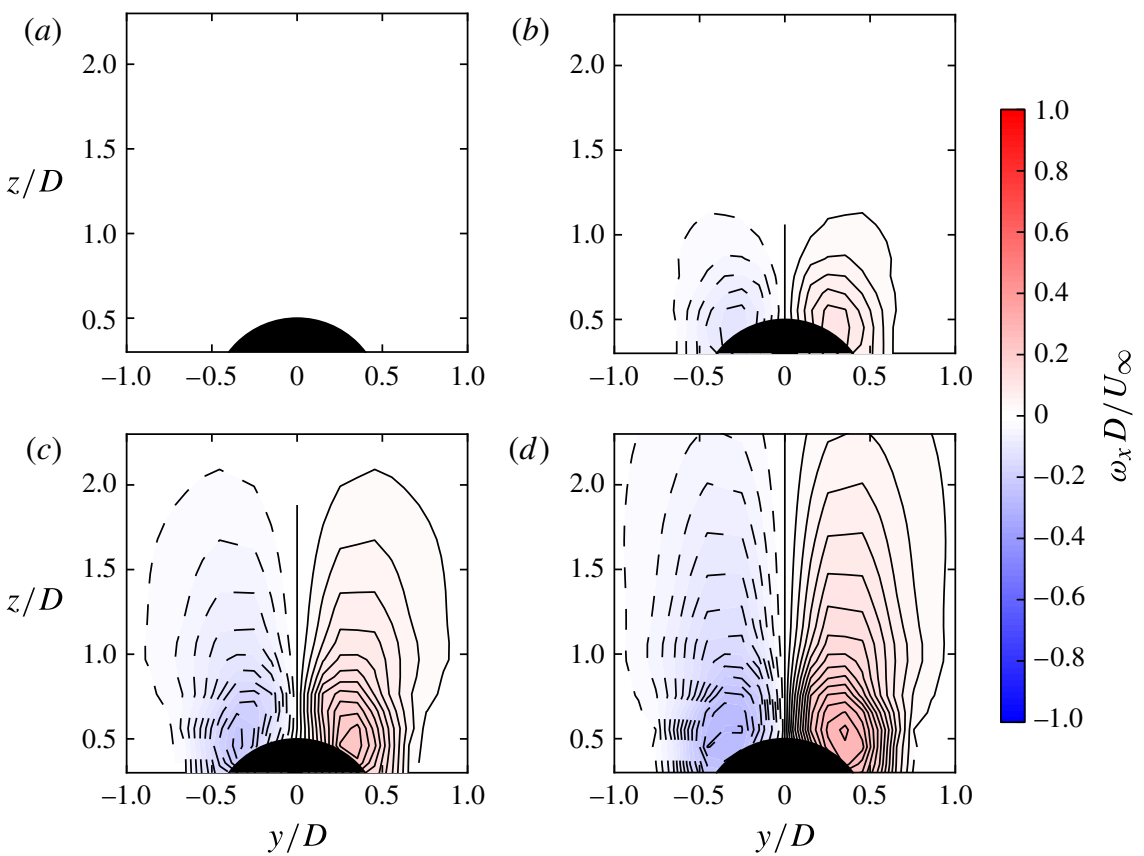

FIGURE 16. Numerical vorticity in the $x$ direction at the back of the cylinder for four yaw angles: (a) $\alpha=0^{\circ}$; (b) $\alpha=3.6^{\circ} ;(c) \alpha=6.3^{\circ} ;(d) \alpha=8.3^{\circ}$. The black lines represent isolines of positive vorticity, and the dashed lines represent isolines of negative vorticity (in steps of 0.02). 
(a)

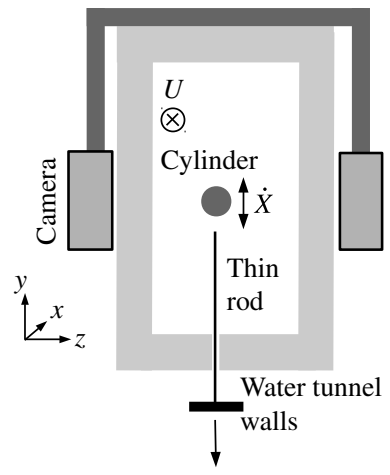

(b)

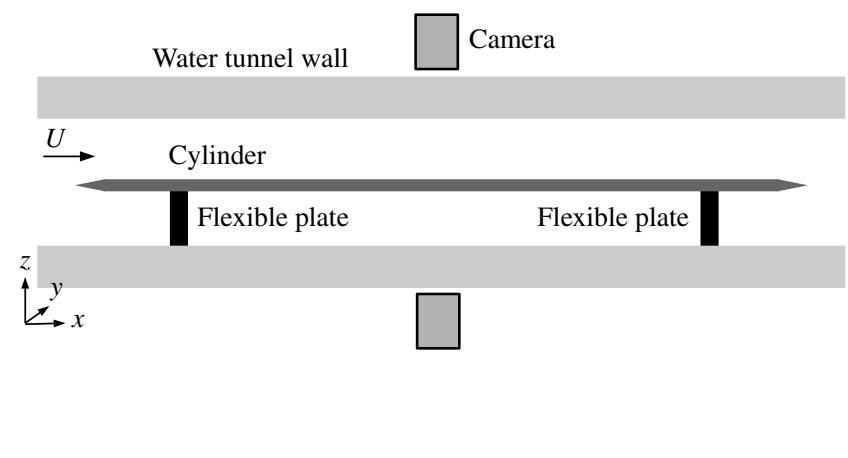

FIGURE 17. Sketch of the water tunnel experiment: $(a)$ view in a lateral plane, showing the thin rod that allows one to set the initial condition for the free oscillations; $(b)$ view in a longitudinal plane.

\section{Dynamic approach: application to the evaluation of the damping}

In this section, we present a dynamic experiment consisting of a cylinder oscillating laterally in a uniform axial flow. The experiment is designed to generate free oscillations of the cylinder. The damping coefficient is deduced from the displacement signal and compared with the damping coefficient derived from the static experiments.

\subsection{Experimental set-up and displacement measurements}

\subsubsection{Geometry and experimental procedure}

The experiment is performed in the test section of a water tunnel. A brass cylinder is placed in the middle of the duct and fixed to one wall of the tunnel with two flexible plates (figure 17). The cylinder has diameter $D=10 \mathrm{~mm}$ and length $L=0.56 \mathrm{~m}$, and its two ends are cone-shaped to avoid flow separation when it is placed in the axial flow. The mass of the cylinder is $0.301 \mathrm{~kg}$. The flexible plates are $25 \mathrm{~mm} \times 10 \mathrm{~mm}$ and have a thickness of $1.0 \mathrm{~mm}$. The water tunnel facility has a cross-section of $S=90 \mathrm{~mm} \times 150 \mathrm{~mm}$. The water flow velocity in the tunnel is varied from 0.5 to $4.0 \mathrm{~m} \mathrm{~s}^{-1}$ with a precision of $0.01 \mathrm{~m} \mathrm{~s}^{-1}$. An initial displacement of $3 \mathrm{~mm}$ is created by pushing and keeping the cylinder aside from its stable equilibrium position with a thin rod passing through a watertight hole in the tunnel wall (see figure 17). Free oscillations of the cylinder with no initial velocity are obtained by pulling abruptly on the thin rod.

The natural frequency of the system in still water is $f=7.74 \mathrm{~Hz}$. When the axial flow velocity is higher than $1 \mathrm{~m} \mathrm{~s}^{-1}$, the absolute value of the instantaneous angle $\alpha(t)=-\dot{X} / U$, where $\dot{X}$ is the cylinder lateral velocity and $U$ is the incoming flow velocity, is always smaller than $5^{\circ}$. The displacement $X$ of the cylinder is measured at its middle using a high-speed and high-precision optical Keyence micrometer composed of a transmission unit which emits light and a receiving unit which detects the position of the shadow of the targeting object. The micrometer has an accuracy of $\pm 0.15 \mu \mathrm{m}$ and a sampling frequency of $400 \mathrm{~Hz}$.

\subsection{Evolution of the frequency and the damping rate with the axial flow velocity}

The time series of the displacement during free oscillations is plotted in figure 18 for two extreme cases of the axial flow velocity, $U=0.7 \mathrm{~m} \mathrm{~s}^{-1}$ and $U=3.9 \mathrm{~m} \mathrm{~s}^{-1}$. 

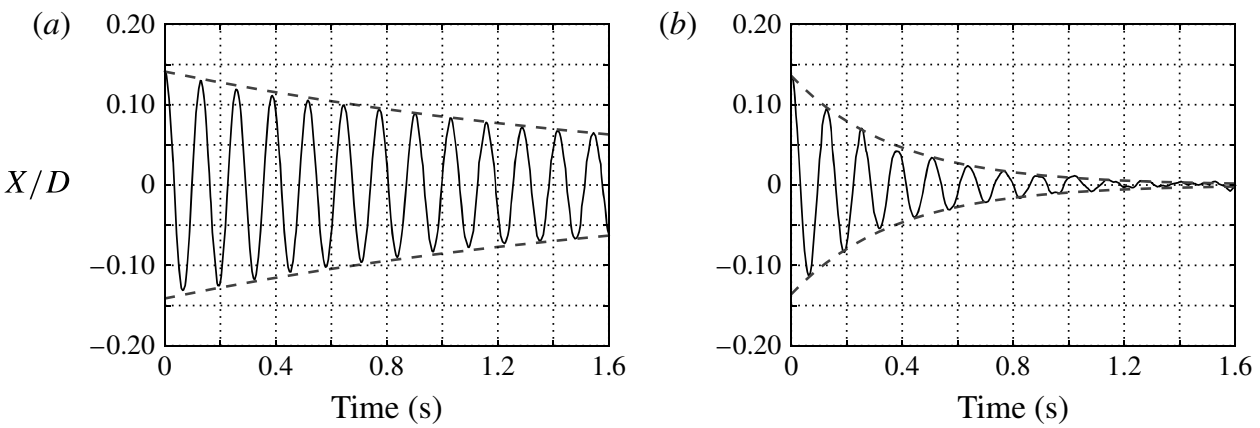

FIGURE 18. Time series of the displacement for two axial flow velocities: $(a) U=$ $0.7 \mathrm{~m} \mathrm{~s}^{-1}(\operatorname{Re}=5800) ;(b) U=3.9 \mathrm{~m} \mathrm{~s}^{-1}(\operatorname{Re}=32500)$.
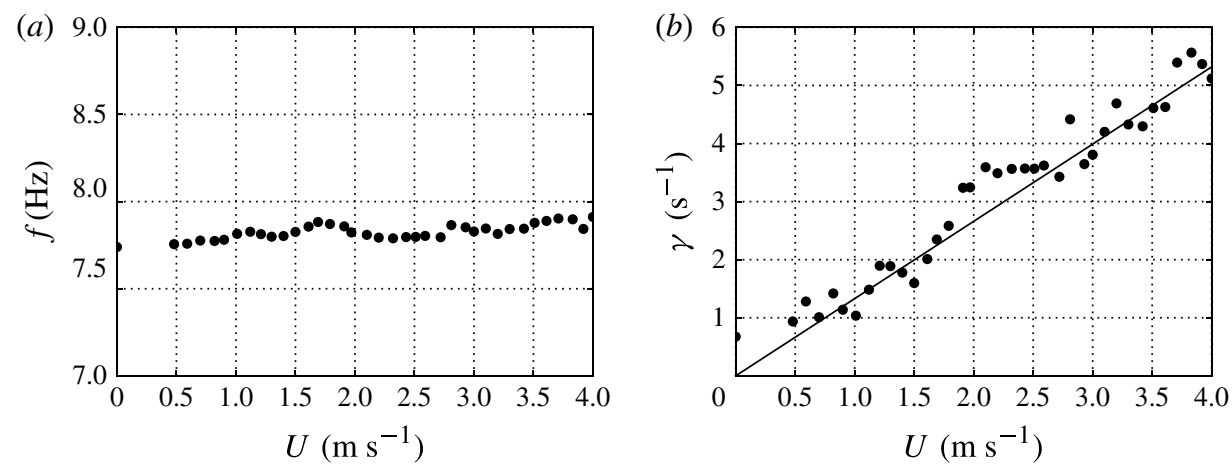

FIgURE 19. Plots versus the axial velocity $U$ of: $(a)$ the mean frequency $f ;(b)$ the mean damping rate $\gamma$.

The envelope clearly decreases according to an exponential law, which allows us to compute a damping rate $\gamma$ by fitting the decay as $\mathrm{e}^{-\gamma t / 2}$. In air, it would take $7.5 \mathrm{~s}$ to get the amplitude halved during a free oscillation; this implies that the structural damping is then negligible compared to the fluid damping.

In practice, the damping-rate calculations are performed with signal amplitudes lower than $2 \mathrm{~mm}$. The oscillation frequency is measured by half-pseudoperiods and remains constant during the free oscillations.

The measurements of oscillation frequency and damping rate are repeated for axial velocities between 0.48 and $4.0 \mathrm{~m} \mathrm{~s}^{-1}$ and presented in figure 19. Figure 19(a) shows that the frequency remains fairly constant, with a slight increase. The damping rate presented in figure $19(b)$ increases linearly with the axial flow velocity as $\gamma=\beta U$ with $\beta=1.33( \pm 0.12) \mathrm{m}^{-1}$.

\subsubsection{Damping coefficient calculation}

It is now supposed that the cylinder displacement satisfies an oscillation equation with a single degree of freedom, where the fluid effects appear as an added mass and an added damping term:

$$
M_{c y l}(1+\mu) \ddot{X}+K_{\text {plates }} X=F_{N} .
$$


Here $M_{c y l}$ represents the cylinder mass, $\mu$ is a mass ratio related to an added mass, $K_{\text {plates }}$ represents the stiffness of the two plates, $X$ denotes the cylinder displacement and $F_{N}$ is the fluid damping. The structural damping is neglected in the present model. It should be noted that eventual added fluid stiffnesses are also neglected in the present approach. Indeed, all forcing terms in phase with the displacement will contribute to the coefficient $\mu$, even if it is due to a possible fluid stiffness.

The observed exponential envelope (see figure 18) suggests that the displacement is the solution of a linear equation, i.e. that $F_{N}$ is linear with respect to the cylinder velocity $\dot{X}$ and can be expressed as

$$
F_{N}=-\frac{1}{2} \rho D L C U \dot{X},
$$

where the damping coefficient $C$ can be calculated from the time displacement of the oscillating cylinder. In dynamics, when the velocity of the cylinder is low compared to the axial flow velocity, the instantaneous angle $\alpha(t)$ is the ratio between the structure and the incoming flow velocities: $\alpha(t)=-\dot{X} / U$. As a consequence, in the free oscillating cylinder experiment, the normal force coefficient or damping force is expressed as

$$
F_{N}=\frac{1}{2} \rho D L C U^{2} \alpha(t),
$$

which is equivalent to the damping force result $C_{N}=C \alpha$ derived from the static experiments in $\S 2.3 .2$.

A measurement of the oscillation frequency in air, $f_{\text {air }}$, is necessary for determining the plate stiffness. In air, no damping is taken into account and the added mass is negligible, which gives $K_{\text {plates }}=M_{c y l}\left(2 \pi f_{\text {air }}\right)^{2}$. The damping coefficient $C$ and the mass ratio $\mu$ presented in (3.4) and (3.5) are obtained by combining (3.1), (3.2) and the expression for the plate stiffness. They are functions of the oscillation frequency of the system in axial flow, the damping rate and the frequency of the system in air:

$$
\begin{aligned}
& C=\frac{M_{c y l}(1+\mu)}{\frac{1}{2} \rho D^{2} L} \frac{D \gamma}{U}, \\
& \mu=\frac{f_{\text {air }}^{2}}{f^{2}+\frac{1}{(2 \pi)^{2}} \frac{\gamma^{2}}{4}}-1 .
\end{aligned}
$$

\subsection{Mass ratio and damping coefficient: comparison with the quasistatic model}

The mass ratio and the damping force coefficient are plotted as functions of the Reynolds number in order to compare the static and dynamic results. With the modelization choice made in (3.1), both quantities depend on the axial flow velocity. An estimation of the mass ratio of the system in still water can be calculated from the measured oscillation frequency in both air and water and the damping rate in water, (3.5). The measured frequency in air is $f_{\text {air }}=8.26 \mathrm{~Hz}$ and decreases to $f=7.74 \mathrm{~Hz}$ in still water. In that case, we find that $\mu=0.136$. In comparison, the ideal inviscid flow analysis of Blevins (1990) predicts that $\mu_{p}=0.142$. As a consequence of the increase in the frequency with the axial flow velocity, there is a $30 \%$ decrease of $\mu$, as observed in figure 20(a). However, this increase of the frequency could also be explained by an added stiffness effect and should then not affect the value of $\mu$. Although the mass ratio influences the damping coefficient (see (3.4)), its variation is too small to have any significant influence. Indeed, as the mass ratio variation is of 

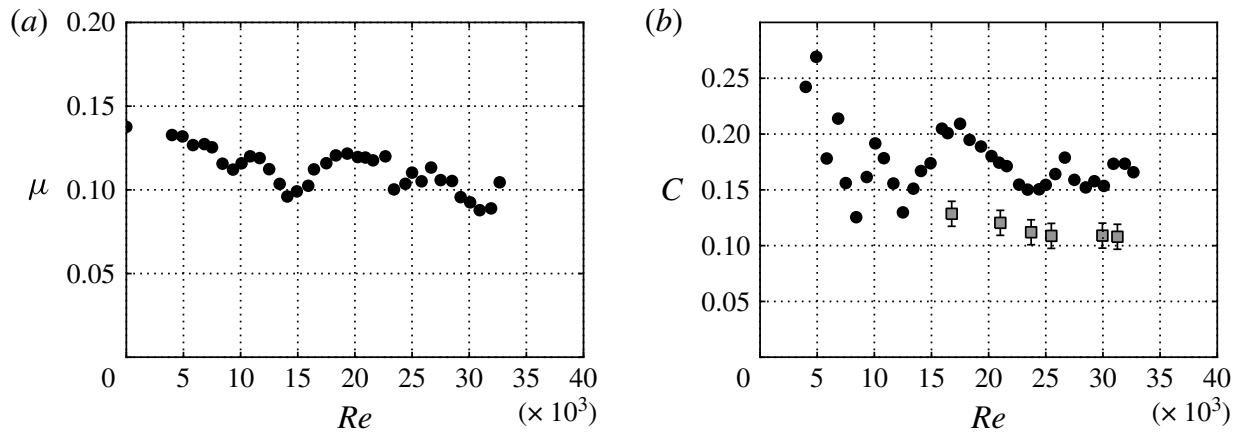

FIGURE 20. Plots versus the Reynolds number $R e$ of: $(a)$ the mass ratio $\mu$; $(b)$ the damping coefficient $C$. The black circles are results from the oscillating cylinder experiment, and the grey squares represent the damping coefficient given by the quasistatic approach.

the order of 0.05 , the maximum variation of the damping induced by the mass ratio is around $5 \%$ only.

Figure $20(b)$ shows the variation of the damping coefficient with the Reynolds number together with the normal force coefficient predicted by the quasistatic approach. The value at $R e=24000$ is the damping coefficient given in (2.7), and the other values are obtained by repeating the static experiment at different Reynolds numbers. For $R e<20000$, the damping coefficient given by the dynamic experiments decreases with the Reynolds number, reaching a constant value $C=0.165 \pm 0.015$. The damping coefficient given by the static approach is $C=0.11 \pm 0.016$. The quasistatic approach is then able to give a good estimation of the damping coefficient in dynamics; the higher damping observed in the dynamic experiment could be due to the flexible plates maintaining the cylinder, since they represent approximately $10 \%$ of the cylinder's projected surface.

\section{Discussion and concluding remarks}

In this article, the fluid forces exerted on a long cylinder in a uniform axial flow have been investigated both statically and dynamically. The normal component of these forces is responsible for the damping of the cylinder oscillating perpendicularly to the flow. If the cylinder velocity is small compared to that of the axial flow, the quasistatic assumption is relevant and study of the fluid force exerted on a static cylinder at small yaw angles is an equivalent problem. The main motivation of this work was to assess the validity of the model proposed by Taylor (1952) to predict the amplitude of the normal forcce at small angles of attack.

The first important result is that, contrary to what is commonly thought, the normal force is dominated by the lift exerted on the cylinder and not by the drag. In fact, the drag contribution is only $10 \%$ of the normal force (figure 8), which renders use of Taylor's model questionable for quantification of the damping forces.

The second important result concerns the linear relationship between the lift force and yaw angles smaller than $5^{\circ}$ (figure 9b). This effect is responsible for the proportionality law between the damping and the axial velocity in the dynamic experiment (figure 19b). The independence principle, which predicts a quadratic dependence, does not hold anymore for small yaw angles. The pressure measurements 
in figure 9 indicate that the lift force originates from the pressure surrounding the cylinder (friction is negligible). In particular, the force is dominated by the front pressure at the dividing streamline, which behaves linearly with respect to the yaw angle. The base pressure, remaining very small, can be considered as a constant equal to the pressure created by the obstruction of the perfectly aligned cylinder (see figure 12). The non-axisymmetry of the pressure distribution is associated with a clear three-dimensional separation that degenerates into a pair of counter-rotating vortices (figure 14). If the vortices are responsible for the constant and weak base pressure, the dividing streamlines are responsible for the linear behaviour of the front pressure. Together, they give rise to the proportionality constant for the damping law with axial flow. We actually find that $C_{p}=0.16 \sin \alpha$. It is worth remarking that numerical simulation using the $k-\omega$ SST turbulence model strengthens the results remarkably. Work still needs to be done to provide a clear explanation for this pressure variation at the front, which would follow a quadratic law if the independence principle were strictly applicable. We believe that theoretical development of an alternative principle applicable to small yaw angles is required.

Further investigations might also include a review of the works that make use of Taylor's model in order to quantify the consequences of the higher damping found in the present article.

\section{REFERENCES}

Archambeau, F., Sakiz, M. \& Namane, M. 2004 Code saturne: a finite volume code for turbulent flows. Intl J. Fin. 1 (1), 1-62.

Blevins, R. D. 1990 Flow-Induced Vibration. Van Nostrand Reinhold.

Bursnall, W. J.\& LofTin, L. K. 1951 Experimental investigation of the pressure distribution about a yawed circular cylinder in the critical Reynolds number range. Tech. Rep. 2463. National Advisory Committee for Aeronautics.

Chen, S. 1987 Flow-induced Vibration of Circular Cylindrical Structures. Hemisphere Publishing Corporation.

Ersdal, S. \& Faltinsen, O. M. 2006 Normal forces on cylinders in near-axial flow. J. Fluids Struct. 22 (8), 1057-1077.

Geuzaine, C. \& Remacle, J.-F. 2009 Gmsh: a three-dimensional finite element mesh generator with built-in pre- and post-processing facilities. Intl J. Numer. Meth. Engng 77 (11), 1309-1331.

Gosselin, F. P. \& DE LANGRe, E. 2011 Drag reduction by reconfiguration of a poroelastic system. J. Fluids Struct. 27 (7), 1111-1123.

Guo, C. Q. \& Paidoussis, M. P. 2000 Stability of rectangular plates with free side-edges in two-dimensional inviscid channel flow. Trans. ASME J. Appl. Mech. 67 (1), 171-176.

Hoerner, S. F. 1965 Fluid-Dynamic Drag: Practical Information on Aerodynamic Drag and Hydrodynamic Resistance. Hoerner Fluid Dynamics.

Hoerner, S. F. 1985 Fluid-Dynamic Lift: Practical Information on Aerodynamic and Hydrodynamic Lift. Hoerner Fluid Dynamics.

Jones, R. T. 1947 Effects of sweepback on boundary layer and separation. Tech. Rep. 884. National Advisory Committee for Aeronautics.

de Langre, E., Païdoussis, M. P., Doaré, O. \& Modarres-Sadeghi, Y. 2007 Flutter of long flexible cylinders in axial flow. J. Fluid Mech. 571, 371-389.

Lighthill, M. J. 1960 Note on the swimming of slender fish. J. Fluid Mech. 9, 305-317.

Menter, F. R. 1993 Zonal two equation k- $\omega$ turbulence models for aerodynamic flows. Tech. Rep. AIAA 93-2906. American Institute of Aeronautics and Astronautics.

Menter, F. R. 1994 Two-equation eddy-viscosity turbulence models for engineering applications. AIAA J. 32 (8), 1598-1605. 
Morison, J. R. \& SchaAf, S. A. 1950 The force exerted by surface waves on piles. Petrol. Trans. AIME 189, 149-154.

Ortloff, C. R. \& IVES, J. 1969 On the dynamic motion of a thin flexible cylinder in a viscous stream. J. Fluid Mech. 38, 713-720.

PAÏDoussis, M. P. 2004 Fluid-Structure Interactions: Slender Structures and Axial Flow, vol. 2. Academic Press, chap. 8 and Appendix Q.

Païdoussis, M. P., Price, S. J. \& DE LANGRe, E. 2011 Fluid-Structure Interactions: Cross-FlowInduced Instabilities. Cambridge University Press.

RAMBERG, S. E. 1983 The effects of yaw and finite length upon the vortex wakes of stationary and vibrating circular cylinders. J. Fluid Mech. 128, 81-107.

Relf, E. H. \& Powell, C. H. 1917 Tests on smooth and stranded wires inclined to the wind direction and a comparison of the results on stranded wires in air and water. Tech. Rep. Reports and Memoranda 307. Aeronautical Research Committee, London.

De Ridder, J., Degroote, J., Van Tichelen, K., Schuurmans, P. \& Vierendeels, J. 2013 Modal characteristics of a flexible cylinder in turbulent axial flow from numerical simulations. J. Fluids Struct. 43, 110-123.

Sears, W. R. 1948 The boundary layer of yawed cylinders. J. Aeronaut. Sci. 15 (1), 49-52.

SMith, R. A., KaO, T. W. \& Moon, W. T. 1971 Experiments on the flow about a yawed circular cylinder. Trans. ASME J. Basic Engng 94 (4), 771-776.

TAYLOR, G. 1952 Analysis of the swimming of long and narrow animals. Proc. R. Soc. Lond. 214, $158-183$.

Zdravkovich, M. M. 2003 Flow Around Circular Cylinders. Volume 2: Applications. Oxford University Press. 